\title{
Avaliação da qualidade de água: concepção hidrossanitária da Bacia do Rio do Campo, Paraná, Brasil
}

O Rio do Campo é responsável por $80 \%$ do abastecimento público do município de Campo Mourão (PR). Sua área compreende, aproximadamente, $170 \mathrm{~km}^{2}$. $\mathrm{O}$ uso do solo no entorno da bacia é predominado pela agricultura, com culturas anuais. $\mathrm{O}$ objetivo deste trabalho foi analisar a qualidade da água por meio dos parâmetros físicos, químicos, microbiológicos e determinação dos índices de qualidade da água (IQA) e estado trófico (IET), da bacia do rio do Campo, além da identificação e quantificação de agroquímicos por cromatografia em fase gasosa acoplada a espectrometria de massas (CG/EM). Foram selecionados 3 pontos de coleta a montante da captação, um ponto no perímetro urbano e dois a jusante. Os resultados obtidos nas análises físico, químico, microbiológico, DBO, nitrogênio e fósforo totais, classificou a água bruta do rio do Campo (na extensão analisada) em função do IQA como ruim e IET como mesotrófico. As análises de 29 agroquímicos por CG/EM, para as amostras de águas coletadas nos seis pontos, mostraram a presença desses analitos em 16 amostras das 30 analisadas, sendo que 17

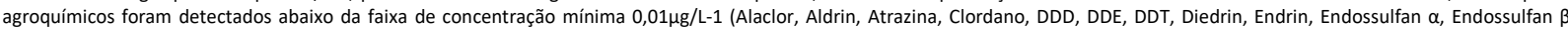
Heptacloro, Heptacloro epóxido, Lindano, Metalocloro, Pendimetalina e Permetrina) e 10 quantificados (Aldrin, DDD, DDE, Diedrin, Endossulfan $\alpha$, Endrin, Heptacloro, Heptacloro epóxido, Metalocloro e Permetrina). A presença destes agroquímicos na bacia do rio do Campo podem comprometer a vida aquática e afetar a saúde humana, uma vez que estes compostos são considerados poluentes orgânicos persistentes com elevado tempo de degradação. Assim, um monitoramento frequente da qualidade da água e das atividades desenvolvidas no entorno devem ser realizadas, para possibilitar maior conservação hídrica.

\section{Water Quality Assessment: Hydrosanitary Conception of the Rio do Campo Basin, Paraná, Brazil}

\begin{abstract}
The Rio do Campo is responsible for $80 \%$ of public supply in the municipality of Campo Mourão (PR). Its area comprises approximately $170 \mathrm{~km}^{2}$. Land use around the basin is predominantly agriculture, with annual crops. The objective of this work was to analyze the water quality through physical, chemical, microbiological parameters and determination of water quality indexes (IQA) and trophic state (IET) of the Campo do basin, as well as the identification and quantification of agrochemicals by gas chromatography coupled to mass spectrometry (GC / MS). Three collection points were selected upstream, one point in the urban perimeter and two downstream. The results obtained in the physical, chemical, microbiological, BOD, nitrogen and total phosphorus analyzes classified the raw water of the Rio do Campo (in the analyzed extension) as a function of the IQA as bad and IET as mesotrophic. Analyzes of 29 agrochemicals by GC/ phosphorus analyzes classified the raw water of the Rio do Campo (in the analyzed extension) as a function of the IQA as bad and IET as mesotrophic. Analyzes of 29 agrochemicals by GC /
MS for water samples collected at the six points showed the presence of these analytes in 16 samples out of 30 analyzed, and 17 agrochemicals were detected below the minimum MS for water samples collected at the six points showed the presence of these analytes in 16 samples out of 30 analyzed, and 17 agrochemicals were detected below the minimum
concentration range $0.01 \mu \mathrm{g} / \mathrm{L}-1$ (Alaclor, Aldrin, Atrazine, Chordane, DDD, DDE, DDT, Diedrin, Endrin, Endosulfan $\alpha$, Endosulfan $\beta$, Heptachlor, Heptachlor Epoxide, Lindane, Metallochlor, concentration range $0.01 \mu \mathrm{g} / \mathrm{L}-1$ (Alaclor, Aldrin, Atrazine, Chordane, DDD, DDE, DDT, Diedrin, Endrin, Endosulfan $\alpha$, Endosulfan $\beta$, Heptachlor, Heptachlor Epoxide, Lindane, Metallochlor,
Pendimethalin and Permethrin) and 10 quantified (Aldrin, DDD, DDE, Diedr, Endosulfan $\alpha$, Endrin, Heptachlor, Heptachlor Epoxide, Metalochlorine and Permethrin). The presence of these agrochemicals in the Campo do Rio basin may compromise aquatic life and affect human health, as these compounds are considered persistent organic pollutants with high degradation time. Thus, frequent monitoring of water quality and surrounding activities should be carried out to enable greater water conservation.
\end{abstract}

Keywords: Environmental control; Water Quality Index; Raw water; Chemical analysis; CG/MS.

Topic: Química Agrícola e Ambiental

Reviewed anonymously in the process of blind peer.
Received: 02/10/2018

Approved: 02/11/2018
Paulo Sergio da Silva

Faculdade Integrado de Campo Mourão, Brasil

http://lattes.cnpq.br/9627324688660184

pssfaculdade@gmail.com

Gilsemara dos Santos Cagni (iD

Centro Universitário de Ensino Superior de Maringá, Brasil http://lattes.cnpq.br/1918081047288115

http://orcid.org/0000-0002-4204-4349

gscagni@hotmail.com

Maria de los Angeles Perez Lizama (in

Centro Universitário de Ensino Superior de Maringá, Brasil

http://lattes.cnpq.br/7827450324471754

http://orcid.org/0000-0002-9714-9383

lizamamdla@hotmail.com
José Eduardo Gonçalves (D)

Centro Universitário de Ensino Superior de Maringá, Brasil

http://lattes.cnpq.br/9921543756032859

http://orcid.org/0000-0002-2505-0536

jose.goncalves@unicesumar.edu.br
Referencing this:

SILVA, P. S.; CAGNI, G. S.; LIZAMA, M. A. P.; GONÇALVES, J. E.. Avaliação da qualidade de água: concepção hidrossanitária da Bacia do Rio do Campo, Paraná, Brasil. Revista Ibero-Americana de Ciências Ambientais, v.9, n.8, p.26-38, 2018. DOI:

http://doi.org/10.6008/CBPC2179-6858.2018.008.0003 


\section{INTRODUÇÃO}

A água é um recurso natural indispensável à manutenção da vida de todas as espécies e para a sobrevivência do homem, sendo considerada uma fonte inesgotável, devido a sua capacidade de purificação e por apresentar um ciclo útil reciclável (VÖRÖSMARTY et al., 2010). Porém, é importante destacar que existe um déficit quando se trata de saneamento (SHANNON et al., 2008). Conforme dados mais recentes da OMS e UNICEF, 663 milhões de pessoas ainda não recebiam água potável até o ano de 2015, um fator preocupante dado à capacidade da água de transmitir doenças (LIU et al., 2017). Em decorrência do crescimento socioeconômico e da necessidade do aumento na produção de alimentos, o homem vem utilizando esse recurso natural, gerando desequilíbrios do meio ambiente e a degradação dos recursos hídricos (FOLEY et al., 2005; STEFFENS et al., 2015; ZHOU et al., 2017).

Diversos fatores podem influenciar a qualidade da água para o consumo, um destes, é o acúmulo de resíduos nos corpos hídricos, demonstrando assim a vulnerabilidade deste recurso natural (ARDILA et al., 2013; STRATHMANN et al., 2016). Outros fatores determinantes são: substrato rochoso, clima, tipo de solo, características físicas da bacia hidrográfica, uso e manejo do solo, cobertura vegetal e atividades desenvolvidas na bacia hidrográfica, sendo estas duas últimas, as que mais alteram a qualidade da água devido à produção desordenada (SILVA et al., 2016).

A quantidade e a qualidade deste recurso são de fundamental importância, e, portanto, é imprescindível a utilização de ferramentas e parâmetros para o monitoramento ao longo dos corpos hídricos. A resolução 357/2005 do CONAMA (CONAMA, 2005) indica os parâmetros de qualidade de água, trazendo também, nesta resolução, a disposição sobre a classificação da água doce quanto ao consumo humano: Classe I, após desinfecção simplificada; Classe II, após tratamento convencional; Classe III sendo águas potáveis, após tratamento convencional ou avançado e a Classe IV que não deve ser destinada ao consumo humano.

O monitoramento dos diversos parâmetros (físicos, químicos e microbiológicos) da qualidade da água ao longo do tempo permite avaliar suas condições de uso e a ocupação do solo em relação à bacia hidrográfica (ANDRIETTI et al., 2016). Diversas ferramentas podem ser utilizadas para demonstrar o uso abusivo e o impacto gerado pelas ações antrópicas nos diversos ecossistemas aquáticos, sendo que o planejamento dos recursos hídricos pressupõe uma distribuição equitativa dos mesmos (BASSO, 2017). Pesquisas atuais demonstram que novas perspectivas estão sendo levantadas sobre o monitoramento da qualidade da água, sendo assim, todos os indicadores contidos nos recursos hídricos devem ser monitorados e considerados em uma análise, para a garantia da sua qualidade (BEZERRA et al., 2013).

A presença de Escherichia coli na água serve como um indicador da qualidade do ambiente. Silva et al. (2016) observou a presença de Escherichia coli no corpo hídrico e concluíram que estava associado ao lançamento de esgotos da área urbana. Outro indicador de qualidade da água pode estar relacionado com o aumento da concentração de elementos químicos, sendo este aumento associado com a precipitação e 
escoamento superficial de sedimentos no corpo hídrico, resultando na elevação de diversos parâmetros como sólidos suspensos totais, turbidez, fósforo, ferro, manganês, cobre, entre outros.

A comunidade fitoplanctônica também é uma ferramenta importante para o monitoramento dos ecossistemas aquáticos, uma vez que esses organismos interferem na qualidade da água, alterando seu gosto, sabor e odor e estão associados à eutrofização. Os nutrientes responsáveis pelo grande crescimento do fitoplâncton são o Nitrogênio (N) e o Fosforo (P) e sua relação com a quantidade de clorofila e a quantidade de fosforo total encontrada na água, influencia diretamente no processo de eutrofização (MAMUN, 2017).

Em estudo realizado na bacia do Rio Pará, Medeiros (2017) demonstrou que, apesar do grande fluxo de água que contribui para a autodepuração do rio, deve ser realizado o monitoramento e o tratamento adequado do esgoto doméstico e industrial na região, bem como, deve-se levar em conta os resíduos armazenados nas bacias (rejeitos) que promovem grandes impactos sobre o rio, trazendo consequências danosas ao ecossistema aquático e a vida da população no entorno.

O monitoramento da qualidade da água, bem como a identificação dos pontos mais vulneráveis à atividade antrópica, deve levar em consideração o gerenciamento, uso, conservação e preservação dos corpos hídricos (SANTI et al., 2012). Atualmente, discutem-se as condições de degradação dos mananciais e nascentes, fundamentais para o abastecimento de importantes rios, bem como a ação nestes recursos naturais (SANTANA, 2017).

No estado do Paraná, o município de Campo Mourão tem, na agricultura, sua principal atividade, utilizando como principal fonte de abastecimento de água, o manancial compreendido pelo rio do Campo e seu entorno. As áreas cortadas pelo mesmo e seus afluentes são utilizadas para exploração agrícola onde se cultiva principalmente soja, milho e trigo, com utilização de grande quantidade de agroquímicos que podem contaminar a água utilizada pela população (SILVA et al., 2016; HOU et al., 2017). Os agroquímicos, notadamente, são poluentes dos corpos hídricos, sendo carreados até estes ambientes pela lixiviação e seu monitoramento, torna-se de extrema importância para o ambiente bem como para a utilização antrópica.

O objetivo deste trabalho visa analisar a qualidade da água por meio dos parâmetros físicos, químicos, microbiológicos e determinação dos índices de qualidade da água (IQA) e estado trófico (IET), da bacia do rio do Campo, no município de Campo Mourão (PR), além da identificação e quantificação de agroquímicos por cromatografia em fase gasosa acoplada a espectrometria de massas (CG/EM), como importante ferramenta para estudos de monitoramento ambiental por esta bacia ser margeada pela urbanização e pela agricultura, dois fatores de grande risco de contaminação.

\section{MATERIAIS E MÉTODOS}

\section{Localização Geográfica da Área de Estudo e Determinação dos Pontos de Coleta das amostras}

O município de Campo Mourão possui uma extensão territorial de $487.730 \mathrm{~km}^{2}$, situando-se na região Sul do Brasil, Centro-Ocidental do estado do Paraná. Possui uma estimativa populacional de 93.547 
habitantes em 2016. Sua localização central está sob as coordenadas - Latitude 24²'37.21"S e Longitude $52^{\circ} 22^{\prime} 41.19 " \mathrm{O}$, e tem sua principal fonte de renda na agricultura (IBGE, 2017).

O clima da região é classificado como Cfa, sendo um clima subtropical úmido mesotérmico, verões quentes e geadas pouco frequentes, tendência de concentrações das chuvas nos meses mais quentes do verão, sem estação de seca definida (ALVARES et al., 2014). A base cartográfica mostra a localização geográfica da Bacia do Rio do Campo e sua posição dentro do perímetro urbano, com localização Municipal e Estadual, conforme a figura 1.
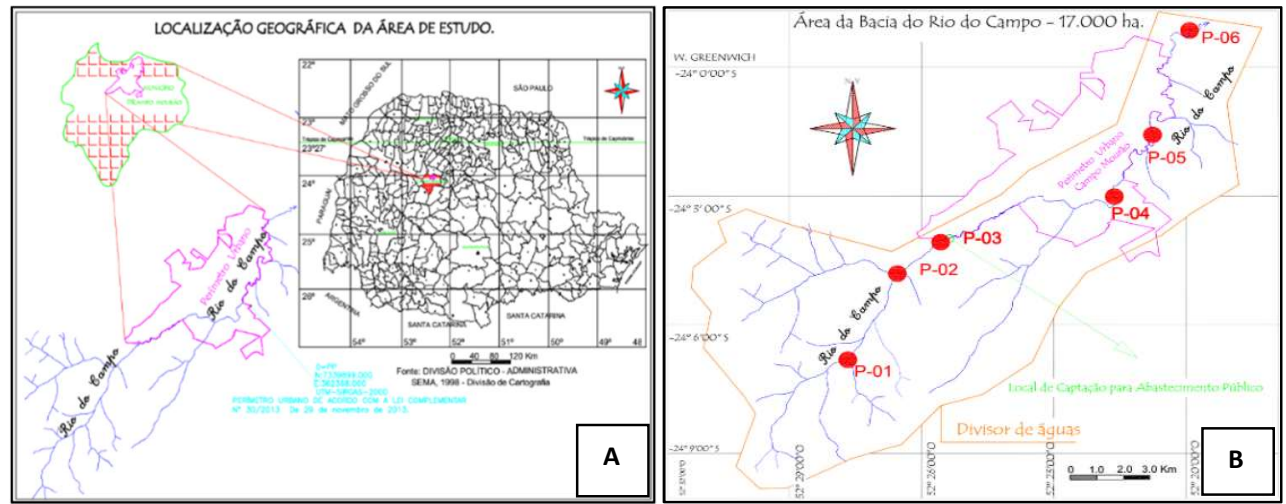

Figura 1: Localização da área de estudo: A) Localização Geográfica; B) Localização da bacia do Rio do Campo, divisor de águas e Pontos de coleta (P-01, P-02, P-03 - pontos a montante do ponto de captação, próximo das nascentes do rio e P-04, P-05, P-06 - pontos a jusante do ponto de captação).

Atualmente, a bacia do Rio do Campo é ocupada por extensão de uso agrícola e parte delimitada ao perímetro urbanizado. As divisas que compõem a captação fluvial, na formação da bacia, são limitadas pelo divisor de águas e a área da bacia detém, aproximadamente, 17.000 hectares (figura 1B). A bacia do Rio do Campo encontra-se identificada no Sistema Geodésico Brasileiro (IBGE, 2017), nas coordenadas $24^{\circ} 6^{\prime} 27.10^{\prime \prime S} ; 52^{\circ} 27^{\prime} 19.91^{\prime \prime O}$, para montante, localizada a sudoeste do perímetro urbano de Campo Mourão, próximo da nascente do rio e $23^{\circ} 59^{\prime} 22.82^{\prime \prime}$; $52^{\circ} 20^{\prime} 2.77 " \mathrm{O}$, para jusante, localizada a nordeste do perímetro urbano de Campo Mourão (figura 1).

Para determinação das coordenadas geográficas, de localização dos pontos de coleta das amostras, utilizou-se a Sistema Global de Posicionamento e de Navegação por Satélite (Global Navigation Satellite System - GNSS). O modelo de equipamento utilizado para verificação ou registro das coordenadas foi Leica Viva GNSS Receptor GS15. Os pontos de coleta selecionados neste estudo, foram identificados através de coordenadas geográficas, como mostra a Tabela 1.

Tabela 1: Planilha de coordenadas geográficas dos pontos de coleta das amostras de água na bacia do Rio do Campo (PR).

\begin{tabular}{|c|c|c|}
\hline Pontos & Latitude & Longitude \\
\hline P1 & $24^{\circ} 6$ '27.10"S & $52^{\circ} 27^{\prime} 19.91^{\prime \prime} \mathrm{O}$ \\
\hline P2 & $24^{\circ} 4^{\prime} 55.10^{\prime \prime S}$ & $52^{\circ} 26^{\prime} 31.83^{\prime \prime} \mathrm{O}$ \\
\hline P3 & $24^{\circ} 4^{\prime} 3.02^{\prime \prime} \mathrm{S}$ & $52^{\circ} 25^{\prime} 26.90^{\prime \prime O}$ \\
\hline P4 & $24^{\circ} 2^{\prime} 52.10^{\prime \prime} \mathrm{S}$ & $52^{\circ} 21^{\prime} 39.32^{\prime \prime} \mathrm{O}$ \\
\hline P5 & $24^{\circ} 1^{\prime} 52.38^{\prime \prime} \mathrm{S}$ & $52^{\circ} 20^{\prime} 51.34^{\prime \prime} \mathrm{O}$ \\
\hline P6 & $23^{\circ} 59^{\prime} 22.82^{\prime \prime} \mathrm{S}$ & $52^{\circ} 20^{\prime} 02.77^{\prime \prime} \mathrm{O}$ \\
\hline
\end{tabular}


Os pontos 01, 02 e 03 estão à montante da captação de água para abastecimento, mais próximos da nascente da bacia do Rio do Campo, composta por sub-bacias. Já, os pontos 04 e 05 estão localizados no perímetro urbano da cidade de Campo Mourão e o ponto 06 localizado na vazante da bacia do Rio do Campo, após uma das estações de tratamento de esgoto da cidade.

\section{Coleta das Amostras de Água}

As amostras de água foram coletadas em frascos estéreis, com tampa e lacre, a uma profundidade de $20 \mathrm{~cm}$ da superfície entre 06 de março de 2016 e 03 de maio de 2017, em período anterior e posterior à aplicação de defensivos agrícola e em seguida armazenada em freezer, para não degradar até o momento de extração e análise. As amostras de água para análises microbiológicas foram conservadas em gelo e analisadas até $24 \mathrm{~h}$ após a coleta. As condições exigíveis para a coleta e a preservação de amostras de água, dos corpos receptores interiores superficiais, seguiram os procedimentos de acordo com as normas da Associação Brasileira de Normas Técnicas (ABNT, 1987).

\section{Caracterização Físicas, Químicas e Microbiológicas das Amostras de Água}

As características físicas e químicas das amostras de água coletadas, tais como, pH, temperatura, condutividade, turbidez, oxigênio dissolvido, sólidos totais dissolvidos, porcentagem de salinidade e potencial de oxidação e redução, foram obtidos pelo equipamento portátil multiparâmetros de qualidade de água Horiba U-50. As concentrações de nitrogênio e fósforo totais, demanda bioquímica de oxigênio e coliformes totais e termotolerantes foram realizados através da metodologia descrita em APHA (2012).

\section{Método de Extração de Resíduos de Agroquímicos em Águas por CG/EM}

As análises de agroquímicos foram realizadas por meio da extração em 1 litro, de amostra de água filtrada, em membrana de $13 \mathrm{~cm}$ de diâmetro e $0,22 \mu \mathrm{m}$ de PVDF (fluoreto de polivinilideno) e, posteriormente, extraídas em funil de separação de 1 litro em três etapas: com uma solução de diclorometano:hexano 50:50 (duas vezes) e com diclorometano (uma vez). Os extratos foram concentrados à temperatura ambiente para um vial de $2 \mathrm{~mL}$.

Os procedimentos de extração foram realizados em triplicata e os resultados foram apresentados como a média dos valores determinados. A determinação do peso seco das amostras foi realizada por gravimetria, para correção do resultado. Para cada lote de amostras foi utilizada uma amostra controle (isenta da matriz), visando controlar qualquer possível interferente decorrente do procedimento de extração.

\section{Condições Cromatográficas para Análise}

As análises foram realizadas em um cromatógrafo a gás (modelo Agilent 7890B) com injetor automático (CTC PAL Control), acoplado a um espectrômetro de massas (modelo Agilent 5977A MSD), equipado com coluna HP-5MS UI Agilent, com fase estacionária de $5 \%$ de fenil metil siloxano $(30,0 \mathrm{~m} \times 250 \mu \mathrm{m}$ x $0,25 \mu \mathrm{m}$ de espessura do filme). Para a separação adequada dos analitos no sistema CG/EM, foi utilizada a 
seguinte programação otimizada de temperatura do forno: temperatura inicial de $50^{\circ} \mathrm{C}$ mantida por 2 min, em seguida rampa de $15^{\circ} \mathrm{C} \mathrm{min}{ }^{-1}$ até $160^{\circ} \mathrm{C}$ mantida por $5 \mathrm{~min}$, e rampa de $5^{\circ} \mathrm{C} \mathrm{min}{ }^{-1}$ até $180^{\circ} \mathrm{C}$, rampa de $10^{\circ} \mathrm{C}$ $\min ^{-1}$ até $270^{\circ} \mathrm{C}$ mantida por $6 \mathrm{~min}$ e finalizando com aumento de $30^{\circ} \mathrm{C} \mathrm{min}-1$ até $300^{\circ} \mathrm{C}$, permanecendo por 1 $\min$.

As demais condições do método de análise foram: volume de injeção de 1,0 $\mu$ l, fluxo do gás de arraste (He, pureza 99,99999\%) igual a $1,0 \mathrm{~mL} \mathrm{~min}^{-1}$ no modo Split 1:5, ionização por impacto eletrônico de $70 \mathrm{eV}$, temperatura da fonte de ionização de $230^{\circ} \mathrm{C}$, do quadrupolo de $150^{\circ} \mathrm{C}$, da linha de transferência de $280^{\circ} \mathrm{C}$ e do injetor de $250^{\circ} \mathrm{C}$. O método do padrão interno foi utilizado para a quantificação em modo monitoramento de íon, selecionado no sistema de detecção EM no modo 'scan' (SIM), na faixa de razão massa/carga ( $m / z)$ de 50 - 550, com 'solvent delay' de 3min, sendo o íon principal utilizado para a quantificação e os íons secundários para a identificação.

Para confirmação da identidade dos agrotóxicos detectados, foi considerado um máximo de $20 \%$ de diferença entre a abundância relativa esperada dos íons secundários em relação ao principal. A aquisição dos dados foi realizada pelo software MassHunter e análise qualitativa para identificação dos compostos foram realizadas por meio da comparação dos espectros de massas dos padrões com os espectros de massas da biblioteca NIST 11.

\section{RESULTADOS}

\section{Análise da Qualidade da Água}

A tabela 2 apresenta os resultados, da qualidade da água nos seis pontos de coleta, ao longo da bacia no Rio do Campo para os seguintes parâmetros: temperatura $\left({ }^{\circ} \mathrm{C}\right)$, potencial hidrogeniônico $(\mathrm{pH})$, potencial de óxido-redução (POR), condutividade $(\Omega)$, oxigênio dissolvido (OD), concentração de oxigênio (\%OD), sólidos Totais (STD) e turbidez.

Tabela 2: Resultados das análises físicas e químicas nas amostras coletadas no período de 06 de março de 2016 a 03 de maio 2017 na bacia do Rio do Campo.

\begin{tabular}{|c|c|c|c|c|c|c|c|c|c|}
\hline Data & coleta & $\mathrm{T}^{\circ} \mathrm{C}$ & $\mathrm{pH}$ & POR & Condutividade $\Omega(\mathrm{mS} / \mathrm{cm})$ & $\begin{array}{c}\text { Turbidez } \\
\text { UNT }\end{array}$ & $\mathrm{OD} \mathrm{mg} / \mathrm{L} \mathrm{O}_{2}$ & $\%$ OD & STD mg/L \\
\hline \multirow[t]{6}{*}{ 06/032016 } & P1 & 15,00 & 7,30 & 233,00 & 0,01 & 57,20 & 10,78 & 108,30 & 9,00 \\
\hline & $\mathrm{P} 2$ & 15,00 & 7,50 & 252,00 & 0,01 & 76,80 & 9,53 & 96,70 & 9,00 \\
\hline & P3 & 14,39 & 7,30 & 269,00 & 0,07 & 70,40 & 10,26 & 102,70 & 4,00 \\
\hline & P4 & 14,14 & 7,00 & 256,00 & 0,03 & 46,20 & 8,35 & 83,70 & 17,00 \\
\hline & P5 & 15,00 & 7,50 & 229,00 & 0,03 & 48,00 & 7,77 & 78,50 & 17,00 \\
\hline & P6 & 14,00 & 7,50 & 159,00 & 0,02 & 90,00 & 8,32 & 88,80 & 10,00 \\
\hline \multirow{6}{*}{$28 / 042016$} & P1 & 16,00 & 6,08 & 321,00 & 0,03 & 83,70 & 11,05 & 127,60 & 20,00 \\
\hline & $\mathrm{P} 2$ & 16,00 & 7,00 & 293,00 & 0,01 & 88,40 & 9,64 & 112,90 & 5,00 \\
\hline & P3 & 17,00 & 7,40 & 229,00 & 0,03 & 54,80 & 10,61 & 114,40 & 18,00 \\
\hline & $\mathrm{P} 4$ & 15,00 & 6,36 & 210,00 & 0,03 & 27,00 & 5,96 & 61,80 & 18,00 \\
\hline & P5 & 16,00 & 7,04 & 184,00 & 0,02 & 138,00 & 12,09 & 129,50 & 11,00 \\
\hline & P6 & 16,50 & 7,00 & 170,00 & 0,02 & 130,00 & 12,00 & 120,00 & 11,00 \\
\hline \multirow{6}{*}{ 09/062016 } & P1 & 15,00 & 7,30 & 246,00 & 0,01 & 36,20 & 8,44 & 100,20 & 7,00 \\
\hline & $\mathrm{P} 2$ & 16,00 & 7,50 & 245,00 & 0,02 & 64,60 & 8,37 & 99,50 & 11,00 \\
\hline & P3 & 16,00 & 7,18 & 214,00 & 0,02 & 31,50 & 7,74 & 91,70 & 9,00 \\
\hline & P4 & 14,14 & 7,46 & 342,00 & 0,01 & 60,80 & 7,46 & 88,70 & 8,80 \\
\hline & P5 & 14,00 & 7,10 & 328,00 & 0,02 & 31,50 & 7,23 & 86,00 & 12,00 \\
\hline & P6 & 15,00 & 7,50 & 322,00 & 0,02 & 32,00 & 18,20 & 95,00 & 14,00 \\
\hline
\end{tabular}




\begin{tabular}{|c|c|c|c|c|c|c|c|c|c|}
\hline & P1 & 18,50 & 7,70 & 277,00 & 0,04 & 122,00 & 4,25 & 51,70 & 28,00 \\
\cline { 2 - 10 } & P2 & 18,50 & 7,50 & 160,00 & 0,07 & 111,00 & 2,37 & 289,00 & 46,00 \\
\cline { 2 - 10 } & P3 & 17,10 & 7,50 & 272,00 & 0,02 & 63,10 & 16,35 & 201,40 & 13,00 \\
\cline { 2 - 10 } & P4 & 18,00 & 7,60 & 291,00 & 0,04 & 64,20 & 11,94 & 146,30 & 24,00 \\
\cline { 2 - 10 } & P5 & 18,10 & 7,50 & 289,00 & 0,01 & 135,00 & 15,11 & 186,40 & 9,00 \\
\cline { 2 - 10 } & P6 & 18,10 & 7,50 & 347,00 & 0,02 & 22,70 & 18,20 & 224,30 & 14,00 \\
\hline \multirow{5}{*}{$03 / 052017$} & P1 & 20,54 & 8,31 & 103,33 & 0,12 & 25,20 & 9,84 & 113,17 & 19,00 \\
\cline { 2 - 10 } & P2 & 19,61 & 7,45 & 154,33 & 0,08 & 29,70 & 9,58 & 107,50 & 20,00 \\
\cline { 2 - 10 } & P3 & 19,64 & 7,19 & 171,75 & 0,03 & 22,70 & 9,31 & 104,55 & 20,00 \\
\cline { 2 - 9 } & P4 & 20,45 & 7,29 & 199,00 & 0,03 & 33,78 & 9,25 & 105,33 & 20,00 \\
\cline { 2 - 9 } & P5 & 20,57 & 7,17 & 182,75 & 0,02 & 31,25 & 8,91 & 101,78 & 20,00 \\
\cline { 2 - 9 } & P6 & 20,96 & 7,29 & 186,00 & 0,09 & 36,43 & 9,46 & 109,05 & 10,00 \\
\hline
\end{tabular}

Fonte: CONAMA (2005); CETESB (2007).

Os resultados de coliformes termotolerantes, DBO, nitrogênio e fósforo totais e cálculos de IQA para os pontos de coleta P3 e P6 são apresentados na tabela 3. É importante notar que estes pontos são indicadores importantes para o índice de qualidade da água, onde o ponto P3 está localizado próximo da captação de abastecimento público e a qualidade da água nesse local deve seguir os parâmetros da legislação, com base na resolução do CONAMA 357 de 2005 (CONAMA, 2005).

O ponto P6 também serve de indicador de qualidade de água, por sua posição estar relacionada ao despejo de cargas de efluente de origens diversas, devido ao rio margear parte do perímetro urbano de Campo Mourão e apresentar uma das estações de tratamento de esgoto, que também deve seguir parâmetros de lançamento de efluente em corpos de água.

Tabela 3: Coliformes Termotolerantes, DBO, Nitrogênio e Fósforo Totais nas amostras coletadas no dia 03 de maio de 2017 e IQA para os pontos P3 e P6 na bacia do Rio do Campo.

\begin{tabular}{|c|c|c|}
\hline Parâmetros- Resolução do Conama 357 & P3 & P6 \\
\hline Coliformes termotolerantes não exceder um limite de 1.000 coliformes por $100 \mathrm{~mL}$ & Sim & Sim \\
\hline DBO 5 dias a $20^{\circ} \mathrm{C}$ até $5 \mathrm{mg} / \mathrm{L} \mathrm{O}_{2}$ & 2,00 & 22,4 \\
\hline Nitrogênio Total $3,7 \mathrm{mg} / \mathrm{L} \mathrm{N}$, para $\mathrm{pH} \leq 7,5$ & 4,25 & 4,25 \\
\hline Fósforo Total Lótico $0,1 \mathrm{mg} / \mathrm{L}$ & 4,25 & 0,10 \\
\hline Potencial hidrogeniônico-pH & 7,19 & 7,29 \\
\hline Temperatura & 19,64 & 20,96 \\
\hline Oxigênio dissolvido & 9,31 & 9,46 \\
\hline Turbidez & 22,70 & 36,43 \\
\hline Resíduo total & 20,00 & 10,00 \\
\hline IQA & 40 & 41 \\
\hline IET(PT) & 77,3 & 57,9 \\
\hline
\end{tabular}

Legenda: IQA = Índice de Qualidade de Água Bruta; IET(PT) - Índice do Estado Trófico para Fósforo total. Fonte: CONAMA (2005); ANA (2016).

Para a otimização do processo de separação, foi preparada uma mistura de 29 agroquímicos orgânicos em água, seguida do procedimento de extração, com os parâmetros cromatográficos e seus respectivos tempos de retenção $\left(t_{R}\right)$. A Tabela 4 mostra os agroquímicos em ordem de eluição, em função do tempo de retenção, os resultados de quantificação obtidos neste trabalho, e os valores máximos permitidos - VMP, encontrados na resolução do CONAMA n 357, de 17 de março de 2005 (CONAMA, 2005). Estes limites também estão de acordo com o padrão europeu (CALDAS et al., 2009) para o controle de resíduos de agroquímicos em água. 
Tabela 4: Parâmetros cromatográficos da mistura de padrões de agrotóxicos analisados por GC/MS, quantificação

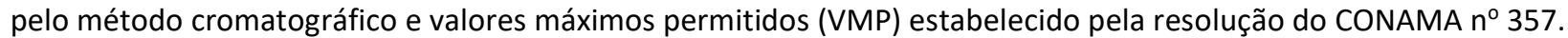

\begin{tabular}{|c|c|c|c|c|}
\hline & Agrotóxicos & $t_{R}$ & Quantificação por GC/MS ( $\left.\mu g L^{-1}\right)$ & $\begin{array}{c}\text { VMP-resolução } 357 \\
\left(\mu \mathrm{L} \mathrm{L}^{-1}\right)\end{array}$ \\
\hline 1 & 1,4-diclorobenzeno & 3,728 & --- & 75 \\
\hline 2 & Naftaleno & 4,809 & --- & --- \\
\hline 3 & Acenafteno & 7,593 & --- & --- \\
\hline 4 & Molinato & 8,369 & --- & 6 \\
\hline 5 & Trifluralina & 11,255 & --- & 20 \\
\hline 6 & Hexaclorobenzeno & 12,085 & & 1 \\
\hline 7 & Simazina & 12,62 & & 2 \\
\hline 8 & Atrazina & 12,85 & & 2 \\
\hline 9 & Lindano & 13,158 & & 2 \\
\hline 10 & Benzo (a)antraceno & 13,451 & --- & 0,018 \\
\hline 11 & Propanil & 15,338 & --- & 20 \\
\hline 12 & Heptacloro & 15,732 & 0,028 & 0,03 \\
\hline 13 & Alaclor & 15,83 & -- & 20,0 \\
\hline 14 & Aldrin & 16,743 & 0,0146 & 0,03 \\
\hline 15 & Metolacloro & 16,887 & 1,115 & 10 \\
\hline 16 & Pendimetalina & 17,87 & & 20 \\
\hline 17 & Heptacloro epóxido & 17,944 & 0,0287 & 0,03 \\
\hline 18 & Clordano & 18,587 & & 0,2 \\
\hline 19 & Endossulfan $\alpha$ & 18,712 & 0,058 & 20 \\
\hline 20 & DDD & 18,919 & 0,026 & 0,02 \\
\hline 21 & Diedrin & 19,32 & 0,0146 & 0,03 \\
\hline 22 & Endrin & 19,804 & 0,0146 & 0,6 \\
\hline 23 & Endossulfan $\beta$ & 20,011 & & 20 \\
\hline 24 & DDT & 20,287 & --- & 0,02 \\
\hline 25 & $\mathrm{DDE}$ & 20,989 & 0,030 & 0,02 \\
\hline 26 & Criseno & 21,972 & & 0,05 \\
\hline 27 & Metoxicloro & 22,128 & & 20 \\
\hline 28 & Permetrina & 24,293 & 0,058 & 20 \\
\hline 29 & Perileno & 26,512 & & 0,018 \\
\hline
\end{tabular}

Fonte: CONAMA (2005).

\section{DISCUSSÃO}

A bacia do Rio do Campo pertence ao manancial de abastecimento do município de Campo MourãoPR e sua captação localizada dentro do perímetro do município, sendo que parte de sua carga hídrica, captada no leito do rio, é utilizada no abastecimento público. Os solos presentes são caracterizados como Latossolo Vermelho, Argissolo Vermelho com textura média e os Nitossolo Vermelho, ocupando respectivamente, $84 \%, 13,6 \%$ e 2,3\% da área (SILVA et al., 2016; EMBRAPA, 2013). Sua ocupação é composta pela agricultura (74\%), com os principais cultivos de soja, milho e trigo, $18 \%$ de vegetação nativa, $7 \%$ de área urbana e 0,5\% de área de pastagem (SILVA et al., 2016).

O Rio do Campo ainda não foi enquadrado nas classes de qualidade, estabelecidas pela Resolução no 357/2005 do CONAMA, desta forma, considera-se este rio de classe II, após tratamento convencional, como estabelecido pela própria resolução (CONAMA, 2005). A temperatura da água do rio do Campo está dentro do esperado para a região e para as datas de coleta (tabela 2), visto que o clima do local de estudo é classificado como $C f a$, subtropical úmido mesotérmico, com geadas pouco frequentes, verões quentes, chuvas concentradas nos meses de verão e sem estação seca definida. A região possui média superior a $25^{\circ} \mathrm{C}$ nos meses mais quentes e inferiores a $15^{\circ} \mathrm{C}$ nos meses mais frios, tendo sua média anual em torno de $20^{\circ} \mathrm{C}$ 
(IAPAR, 2012). Com isso, a variação da temperatura da água ao longo das datas é inerente à temperatura do ambiente.

A água, destinada ao consumo humano, deve possuir pH na faixa de 6,0 a 9,5, de acordo com a resolução do CONAMA 357/2005. Neste estudo foram observados valores dentro do exigido pela legislação, contudo, em algumas amostras houve redução nos valores de pH (tabela 2), possivelmente devido à presença de matéria orgânica na água (CONAMA, 2005).

Ao observar os valores de turbidez (tabela 2) é possível notar que nos dias 28/04/2016, nos pontos P5 e P6, e em 19/06/2016, nos pontos P1, P2 e P5, ocorreu um possível carreamento de matéria orgânica no corpo hídrico, ocasionando o aumento da turbidez da água, uma vez que nesta data houve um grande volume de chuva. Estudo realizado na bacia do Rio do Campo por Carvalho et al. (2015) apresentou resultado semelhante para o ponto P6. A presente similaridade ocorreu devido ao fato que o mês de março possui um volume maior de chuva, ocorrendo assim, transporte de material particulado das terras agricultáveis do entorno, para o corpo hídrico.

Todos os valores de resíduos sólidos totais dissolvidos ficaram abaixo do máximo permitido pela resolução do CONAMA 357/2005, comportamento semelhante ao observado no trabalho de Carvalho et al. (2015). Os maiores valores de sólidos totais dissolvidos STD, foram observados nos pontos P1 e P2, no dia 19/06 (tabela 2). A ocorrência desses valores elevados, em relação aos demais pode estar relacionada ao transporte de resíduos para o leito do rio.

Os resultados de nitrogênio obtidos para os pontos P3 e P6 apresentaram valores 13\% acima do máximo permitido pela legislação, que é de $3,7 \mathrm{mg} \mathrm{L}^{-1}$, enquanto o fósforo, no ponto $\mathrm{P} 3$, apresentou uma concentração 45 vezes maior que o permitido pela resolução do CONAMA 357/2005 (tabela 3). Por meio destes dados é possível classificar a qualidade da água (IQA) nos pontos de coleta P3 e P6 como péssima (CONAMA, 2005).

O ponto P3 sofre influência direta das áreas agrícolas existentes na bacia do Rio do Campo, dessa forma, esses elementos podem ser atribuídos à utilização de fertilizantes nitrogenados e fosfatados que são aplicados no solo e que durante o ciclo produtivo de culturas como soja, milho e trigo, por meio do escoamento superficial da água sejam levados para o leito do rio, juntamente com o solo. Esse fenômeno também pode estar associado à eutrofização, consequentemente com o aumento da $\mathrm{DBO}$, como observado no ponto P6. Os valores elevados de nitrogênio e fósforo, identificados nos pontos P3 e P6 podem indicar também a decomposição da matéria orgânica presente na água.

Santos et al. (2014) concluiu em seu trabalho que o elevado nível de fósforo, presente no açude de Orós (CE), era devido à ausência de práticas conservacionistas como terraceamento nas áreas agrícolas, ocorrendo assim uma diminuição da infiltração de água de chuva, provocando o escoamento superficial, o carreamento de solo e seus elementos nutricionais, bem como a porção de matéria orgânica, para o manancial. Lopes et al. (2007) identificou que, quando atividades antrópicas são realizadas nos solos de bacias hidrográficas, sem práticas de manejo, a qualidade da água sofre impactos negativos. Bicudo et al. 
(2010) cita que entre os impactos estão o aumento dos sedimentos, redução na concentração de oxigênio e aceleração do assoreamento.

O ponto P6 localiza-se a jusante da estação de tratamento de esgoto da cidade de Campo Mourão$\mathrm{PR}$, dessa forma os resultados indicam que a elevada taxa de DBO e nitrogênio podem ser atribuídos à presença de esgoto doméstico na água, explicando também os resultados de coliformes termololerantes neste ponto (MATOS, 2005). Outro fator importante atribuído aos níveis elevados de nitrogênio e DBO neste ponto de coleta, pode estar relacionado à ineficiência ou sobrecarga no sistema de tratamento de esgoto do município de Campo Mourão, visto que ele recebe a maior parte de carga de dejetos da cidade e que, em determinados momentos, pode ser sobrecarregado devido ao excesso de chuvas.

Com base nestes resultados (tabela 3), foi possível determinar o índice de qualidade de água (IQA) para o Rio do Campo, na extensão compreendida entre os pontos P3 e P6, que vai desde a captação até a jusante da estação de tratamento. Para este trecho, a água bruta do Rio de Campo apresentou IQA de 41, sendo classificada como ruim (ANA, 2016). O IQA é um parâmetro muito importante para a classificação dos corpos de água, apontando o nível de tratamento a ser realizado de acordo com a classe atribuída ao rio. Desta forma, este indicador de qualidade de água é uma importante ferramenta para classificar adequadamente o Rio do Campo e não atribuir simplesmente classe nível 2, devido a falta de dados sobre a qualidade da água bruta deste rio.

A presença em excesso de nitrogênio e fósforo nos rios, promove o processo de eutrofização, que ocasiona a proliferação de macrófitas e algas planctônicas (WANG et al., 2009; HOU et al., 2016), o que leva a contaminação dos rios devido às substâncias tóxicas liberadas pelas algas, redução do oxigênio e morte dos peixes (VIANA et al., 2009), além de elevar o custo de tratamento desta água. Estima-se que cerca de $41 \%$ dos lagos e reservatórios da América do Sul tenham problemas de eutrofização devido à ação antrópica (NYENJE et al., 2010).

A determinação do IET (PT) nos pontos de coleta P3 e P6 (tabela 3) foi de 77,3 e 57,9, respectivamente. Estes valores apontam para a classificação do IET como sendo mesotrófico (ANA, 2016; CETESB, 2007; LAMPARELLI, 2004), apresentando corpos d'água com produtividade intermediária, com possíveis implicações sobre a qualidade da água, mas em níveis aceitáveis, na maioria dos casos.

Em virtude da variabilidade sazonal dos processos ambientais que têm influência sobre o grau de eutrofização de um corpo hídrico, esse processo pode apresentar variações no decorrer do ano, havendo épocas em que se desenvolve de forma mais intensa e em outras moderadamente (ANDRIETTI et al., 2016). Em geral, no início da primavera, com o aumento da temperatura da água, há uma maior disponibilidade de nutrientes e condições propícias de penetração de luz na água, sendo comum observar-se um incremento do processo, ao passo que no período de inverno, esse processo se mostra menos intenso. Nesse sentido, a determinação do grau de eutrofização médio anual de um corpo hídrico, pode não identificar, de forma explícita, as variações que ocorreram ao longo do período anual, uma vez que não é possível apresentar os resultados mensais para cada ponto amostral. 
As condições cromatográficas, aplicadas no desenvolvimento deste trabalho, foram validadas para a análise multirresíduos de agroquímicos por SABIN et al. (2009), onde foi descrito o método de extração e análise por CG/EM. A seletividade do método aplicada neste estudo mostrou-se adequada para a determinação de agroquímicos na água bruta do rio. Para análises de rotina foram utilizadas curvas analíticas, em três concentrações: $0,010,0,100$ e 0,300 $\mathrm{g} \mathrm{L}^{-1}$, visando à identificação e quantificação dos mesmos, em comparação com os valores permitidos pela legislação vigente (CONAMA, 2005).

Todos os agroquímicos descritos na tabela 4 foram separados e quantificados, uma vez que os mesmos apresentaram alta sensibilidade e adequada recuperação na extração multirresíduos. Neste estudo, a linearidade foi adequada para todos os compostos. A aplicação do método, com amostras obtidas nos pontos de coleta do Rio do Campo, mostrou excelente desempenho em termos de adequação do sistema, na faixa de concentração, entre 0,010 e $0,300 \mu \mathrm{L} \mathrm{L}^{-1}$, mostrando ser possível na utilização em análises de rotina para qualquer tipo de amostra de água.

Os resultados das análises dos agroquímicos, nas amostras de águas coletadas nos seis pontos, mostraram a presença desses analitos em 16 amostras das 30 analisadas. Dos 29 agroquímicos analisados

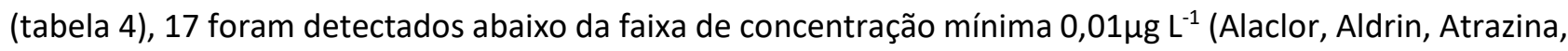
Clordano, DDD, DDE, DDT, Diedrin, Endrin, Endossulfan $\alpha$, Endossulfan $\beta$, Heptacloro, Heptacloro epóxido, Lindano, Metalocloro, Pendimetalina e Permetrina) e 10 quantificados (Aldrin, DDD, DDE, Diedrin, Endossulfan $\alpha$, Endrin, Heptacloro, Heptacloro epóxido, Metalocloro e Permetrina). Para a quantificação, foram utilizados os compostos que apresentaram concentrações acima do valor mínimo de $0,01 \mu \mathrm{g} \mathrm{L}^{-1}$.

O DDT não foi quantificado nas amostras, porém, foi possível quantificar seus metabólitos, DDD e o DDE. O DDD foi quantificado em uma amostra com $0,026 \mu \mathrm{L} \mathrm{L}^{-1}$, já o DDE foi encontrado em 12 amostras e quantificado em 7 delas, com concentração média de $0,030 \mu \mathrm{L} \mathrm{L}^{-1}$, comprovando que é o metabólito mais estável.

O heptacloro sofre epoxidação tornando-se o heptacloro epóxido, pertencente à classe toxicológica II, altamente tóxico, apresentando uma longa persistência no ambiente. O heptacloro epóxido, a exemplo de seu precursor, apresentou concentrações de $0,0287 \mu \mathrm{g} \mathrm{L}^{-1}$. O valor determinado na análise por CG/EM está no limite do valor máximo permitido, estipulado pela resolução do CONAMA nº 357 (CONAMA, 2005), que é de $0,030 \mu \mathrm{g} \mathrm{L}^{-1}$. O endrin é um inseticida pertencente à classe toxicológica I, usado principalmente nas culturas de cana-de-açúcar, soja, algodão e milho. Este possui efeitos tóxicos similares ao aldrin e dieldrin, mas é

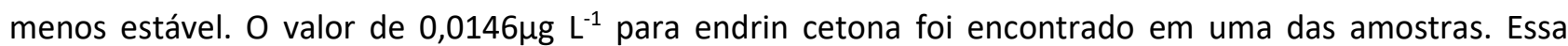
substância não está entre os agroquímicos relacionados pela portaria no 2.914/2011 (BRASIL, 2011), que estabelece valor máximo de $0,600 \mu \mathrm{g} \mathrm{L}^{-1}$ apenas para o endrin.

Com base nas análises de CG/EM realizadas, foi possível detectar a presença de compostos organoclorados, tais como, Aldrin, DDT, Endossulfam, Endrin, Heptacloro, Metolacloro e Permetrina nos diferentes pontos e datas de coletas realizadas no Rio do Campo. A contaminação deste corpo d'água pode estar relacionada ao uso desses agroquímicos, nos períodos em que houve a colonização agrícola da região 
da bacia do Rio do Campo (PR), uma vez que são considerados poluentes orgânicos persistentes e muito deles apresentam um elevado tempo de degradação.

\section{CONCLUSÕES}

As análises físicas, químicas e microbiológicas realizadas neste estudo, aliados aos demais parâmetros abordados como: DBO, nitrogênio e fósforo totais, os valores obtidos de IQA e IET podem levar a classificar a água bruta do Rio do Campo como ruim e o estado de trofia como mesotrófico. A técnica de CG/EM demonstrou ser fundamental para determinações que exigem extrema sensibilidade e seletividade, atendendo a requisitos na faixa de $\mu \mathrm{g} \mathrm{L}^{-1}$ para todos os compostos e apresentando seletividade para possibilitar determinações de resíduos de agroquímicos, em amostras contendo parâmetros variados, inclusive com alta turbidez, sem interferências significativas.

O método permitiu identificar 17 agroquímicos organoclorados nas amostras de água coletada no rio do Campo. Os limites encontrados foram baixos, suficientes para se detectar essas substâncias a um nível inferior ao estabelecido pela legislação nacional. Como o uso do solo no entorno da bacia do Rio do Campo é explorado pela agricultura, as consequências das ações antrópicas são preocupantes em relação à qualidade da água disponível, pois se trata de um manancial de captação para abastecimento público e sua preservação, deveria ser realizada de forma mais ampla.

\section{REFERÊNCIAS}

ABNT. Associação Brasileira de Normas Técnicas. NBR 9898: Preservação e técnicas de amostragem de efluentes líquidos e corpos receptores. Rio de Janeiro: ABNT, 1987.

ALVARES, C. A.; STAPE, J. L.; SENTELHAS, P. C.; GONÇALVES, J. L. M.; SPAROVEK, G.. Köppen's climate classification map for Brazil. Meteorologische Zeitschrift, v.22, n.6, p.711-728, 2014. DOI: http://doi.org/10.1127/0941-2948/2013/0507

ANA. Agência Nacional de Águas. Portal da Qualidade das Águas. Brasília: ANA, 2016.

ANDRIETTI, G.; FREIRE, R.; AMARAL, A. G.; ALMEIDA, F. T.; BONGIOVANI, M. C.; SCHNEIDER, R. M.. Índices de qualidade da água e de estado trófico do rio Caiabi, MT. Revista Ambiente e Água, v.11, n.1, p.162-175, 2016. DOI: http://doi.org/10.4136/1980-993X

APHA. American Public Health Association. Standard Methods for examination of water and wastewater. 22 ed. Washington: APHA, 2012.

ARDILA, A. N.; SALDARRIAGA, J. C.. Formulación de un Índice Global de Calidad de Aguas Residuales para Riego. Avances en Ciencias e Ingeniería, v.4, n.3, p.11-26, 2013.

BASSO, E. R.; CARVALHO, S. L.. Avaliação da qualidade da água em duas represas e uma lagoa no município de llha Solteira (SP). Holos Environment, v.7, n.1, p.16-29, 2017.

BEZERRA, J. M.; SILVA, P. C. M.; BATISTA, R. O.; PINTO, C. H. C.; FEITOSA, A. P.. Análise dos indicadores de qualidade da água no trecho urbano do Rio Apodi-Mossoró em Mossoró-
RN, Brasil. Semina: Ciências Agrárias, v.1, n.34, p.3443-3454, 2013. DOI: http://doi.org/10.5433/16790359.2013v34n6Supl1p3443

BICUDO, C. E. M.; TUNDISI, J. G.; SCHEUENSTUHL, M. C. B. Águas do Brasil: Análises Estratégicas. São Paulo: Instituto de Botânica, 2010.

BRASIL. Ministério da Saúde. Portaria n.2914 de 12 de dezembro de 2011. Brasília: MS, 2011.

CARVALHO, K. Q.; LIMA, S. B.; PASSING, F. H.; GUSMÃO, L. K.; SOUZA, D. C.; KREUTZ, C.; BELINI, A. D.; ARANTES, E. J.. Influence of urban area on the water quality of the Campo River basin, Paraná State, Brazil. Brazilian Journal of Biology, v.75, n.4, p.96-106, 2015. DOI: http://doi.org/10.1590/1519-6984.00413suppl

CETESB. Companhia Ambiental do Estado de São Paulo. Relatório de Qualidade das Águas Interiores no Estado de São Paulo: 2006. São Paulo: CETESB, 2007.

CONAMA. Conselho Nacional do Meio Ambiente. Resolução n.357 de 17 de março de 2005. Dispõe sobre a classificação dos corpos de água e diretrizes ambientais para o seu enquadramento, bem como estabelece as condições e padrões de lançamento de efluentes, e dá outras providencias. Brasília: DOU, 2005.

EMBRAPA. Empresa Brasileira de Pesquisa Agropecuária. Sistema brasileiro de classificação de solos. 3 ed. Brasília: EMBRAPA, 2013. 
FOLEY, J. A.; DEFRIES, R.; ASNER, G. P.; BARFORD, C.; BONAN, G.; CARPENTER, S. R.; CHAPIN, F. S.; COE, M. T.; DAILY, G. C.; GIBBS, H. K.; HELKOWSKI, J. H.; HOLLOWAY, T.; HOWARD, E. A.; KUCHARIK, C. J.; MONFREDA, C.; PATZ, J. A.; PRENTICE, I. C.; RAMANKUTTY, N.; SNYDER P. K.. Global consequences of land use Science. Science, v.309, p.570-574, 2005.

HOU, W.; SUN, S.; WANG, M.; LI, X.; ZHANG, N.; XIN, X.; SUN, L.; LI, W.; JIA, R.. Assessing water quality of five typical reservoirs in lower reaches of Yellow River, China: Using a water quality index method. Ecological Indicators, v.61, p.309-316, 2016. DOI:

https://doi.org/10.1016/j.ecolind.2015.09.030

IAPAR. Instituto Agronômico do Paraná. Cartas climáticas: Classificação climática. Curitiba: IAPAR, 2012.

IBGE. Instituto Brasileiro de Geografia e Estatística. Informações sobre os municípios brasileiros. Brasília: IBGE, 2017.

IBGE. Instituto Brasileiro de Geografia e Estatística. Projeto Mudança do Referencial Geodésico - PMRG. Brasília: IBGE, 2005.

LAMPARELLI, M. C.. Grau de trofia em corpos d'água do Estado de São Paulo: avaliação dos métodos de monitoramento. Tese (Doutorado em Biociências) Universidade de São Paulo, São Paulo, 2004.

LOPES, F. W. A.; DUTRA, G. C.; PEREIRA, J. A. A.; CARVALHO, L. M. T.. Avaliação da influência de áreas de solo exposto sobre a qualidade das águas do Ribeirão de Carrancas-MG. In: SIMPÓSIO BRASILEIRO DE SENSORIAMENTO REMOTO, 13. Anais. São José dos Campos: INPE, 2007.

MAMUN, M.; AN, K.. Major nutrients and chlorophyll dynamics in Korean agricultural reservoirs along with an analysis of trophic state index deviation. Journal of AsiaPacific Biodiversity, v.10, n.2, p.183-191, 2017. DOI: https://doi.org/10.1016/j.japb.2017.04.001

MATOS, A. T.; EMMERICH, I. N.; BRASIL, M. S.. Tratamento por escoamento superficial de águas residuárias da lavagem e despolpa dos frutos do cafeeiro. Engenharia na Agricultura, v.3, n.4, p.240-246, 2005. DOI: http://doi.org/10.1590/S0100-69162011000100016

MEDEIROS, A. C.; FAIAL, K. R. F.; FAIAL, K. C. F.; LOPES, I. D. S.; LIMA, M. O.; GUIMARÃES, R. M.; MENDONÇA, N. M.. Quality index of the surface water of Amazonian rivers in industrial areas in Pará, Brazil. Marine Pollution Bulletin, v.123, n.1, p.156-164, 2017. DOI:

https://doi.org/10.1016/j.marpolbul.2017.09.002

NYENJE, P. M.; FOPPEN, J. W.; UHLENBROOK, S.; KULABAKO, R.; MUWANGA, A.. Eutrophication and nutrient release in urban areas of sub-Saharan Africa-A review. Science of the
Total Environment, v.408, n.3, p.447-455, 2010. DOI: http://doi.org/10.1016/i.scitotenv.2009.10.020

SANTANA, N. R. F.; AGUIAR NETO, A. O.; SILVA, M. G.; GARCIA, C. A. B.. Índice de qualidade da água nas nascentes do Rio Piauitinga-SE por análise multivariada e o uso na irrigação. Revista Brasileira de Agricultura Irrigada, v.10, n.6, p.999-1010, 2017. DOI: http://doi.org/10.7127/rbai. v10n600441

SANTI, G. M.; FURTADO, C. M.; MENEZES, R. S.; KEPPELER, E. C.. Variabilidade espacial de parâmetros e indicadores de qualidade da água na sub-bacia hidrográfica do Igarapé São Francisco, Rio Branco, Acre, Brasil. Ecologia Aplicada, v.11, n.1, p.23-31, 2012.

SANTOS, J. C. N.; ANDRADE, E. M.; ARAÚJO NETO, J. R.; MEIRELES, A. C. M.; PALÁCIO, H. A. Q.. Land use and trophic state dynamics in a tropical semi-arid reservoir. Revista Ciência Agronômica, v.45, n.1, p.35-44, 2014. DOI: http://doi.org/10.1590/S1806-66902014000100005

SILVA, V. B.; GASPARETTO, N. V. L.. Qualidade da água na sub-bacia do Rio do Campo - Campo Mourão-PR. Revista Brasileira de Geografia Física, v.9, n.2, p.585-600, 2016.

STRATHMANN, M.; HORSTKOTT, M.; KOCHC, C.; GAYERC, U.; WINGENDERBA, J.. The River Ruhr: an urban river under particular interest forrecreational use and as a raw water source for drinking water: Thecollaborative research project "Safe Ruhr": microbiological aspects. International Journal of Hygiene and Environmental Health, v.219, p.643-661, 2016. DOI: https://doi.org/10.1016/j.ijheh.2016.07.005

VIANA, R. B.; CAVALCANTE, R. M.; BRAGA, F. M. G.; VIANA, A. B.; ARAÚJO, J. C.; NASCIMENTO, R. F.; PIMENTEL, A. S.. Risk assessment of trihalomethanes from tap water in Fortaleza, Brazil. Environmental Monitoring and Assessment, v.151, p.317-325, 2009. DOI: https://doi.org/10.1007/s10661-008-0273-y

VÖRÖSMARTY, C. J.; MCINTYRE, P. B.; GESSNER, M. O.; DUDGEON, D.; PRUSEVICH, A.; GREEN, P.; GLIDDEN, S.; BUNN, S. E.; SULLIVAN, C. A.; LIERMANN, C. R.; DAVIES, P. M.. Global threats to human water security and river biodiversity. Nature, v.467, n.7315, p.555-561, 2010. DOI: http://doi.org/10.1038/nature09440

WANG, H.; WANG, H.. Mitigation of lake eutrophication: loosen nitrogen control and focus on phosphorus abatement. Progress in Natural Science, v.19, p.1445-1451, 2009. DOI: https://doi.org/10.1016/j.pnsc.2009.03.009

ZHOU, Y.; MA, J.; ZHANG, Y.; QIN, B.; JEPPESEN, E.; SHI, K.; BROOKESF, J. D.; SPENCERG, R. G. M.; ZHU, G.; GAO, G.. Improving water quality in China: Environmental investment pays dividends. Water Research, v.118, n.1, p.152-159, 2017. DOI: https://doi.org/10.1016/j.watres.2017.04.035

A CBPC - Companhia Brasileira de Produção Científica (CNPJ: 11.221.422/0001-03) detém os direitos materiais desta publicação. Os direitos referem-se à publicação do trabalho em qualquer parte do mundo, incluindo os direitos às renovaç̃̃es, expansões e disseminações da contribuiç̃o, bem como outros direitos subsidiários. Todos os trabalhos publicados eletronicamente poderão posteriormente ser publicados em coletâneas impressas sob coordenação da Sustenere Publishing da Companhia Brasileira de Produção Científica e seus parceiros autorizados. Os (as) autores (as) posteriormente ser publicados em coletâneas impressas sob coordenação da Sustenere Publishing, da Companhia Brasileira de Produção Cientifica e seus pã 